$4^{\text {th }}$ International Conference on Industrial Engineering and Industrial Management

XIV Congreso de Ingeniería de Organización

Donostia - San Sebastián, September $8^{\text {th }}-10^{\text {th }} 2010$

\title{
Sistema experto basado en la lógica difusa para la detección de configuraciones climáticas asociadas al confort
}

\author{
Expert system based on fuzzy logic to detect configurations associated \\ to climatic comfort
}

\author{
Pablo Aparicio-Ruiz, Joaquín R. Fernández Valverde y Luis Onieva Giménez \\ Dpto. de Organización Industrial y Gestión de Empresas. \\ Escuela Técnica Superior de Ingenieros. Universidad de Sevilla, Camino de los Descubrimientos, s/n, 4 I 092 Sevilla. \\ pabloaparicio@us.es jfernandez9@us.es Onieva@esi.us.es
}

Fecha de recepción: 8-9-2010

Fecha de aceptación: 27-10-2010

Resumen: A través de la lógica difusa es posible detectar patrones de comportamiento que generalmente ocurren en un edificio, en el caso de los sistemas de climatización, se pueden detectar situaciones en las que decidir entre maximizar el confort y/o maximizar el ahorro. Este artículo presenta una metodología para el ahorro de energía en estos sistemas, en función de la ocupación y la sensación de confort de sus ocupantes. Esta metodología proporciona información importante y útil para la selección del punto de ajuste del confort de las salas de un sistema de climatización central, sin necesidad de utilizar valores fijos basados en los programas horarios u otras metodologías.

Palabras clave: lógica difusa, confort, sistema experto.

Abstract: Through fuzzy logic it is possible to detect behavior patterns that generally occur in a building, in the case of HVAC (Heating, Ventilating and Air Conditioning) systems, can be detected situations in which to decide between maximizing comfort and maximizing savings. This paper presents a methodology in order to energy savings in HVAC systems of buildings, depending on the occupation and the feeling of comfort that their occupants shown. This methodology provides important and useful information for selecting the set point of rooms comfort and adapting the power of the central HVAC system of the building, without any need of using fixed values based on program schedules or other methodologies.

Keywords: fuzzy logic, human comfort, expert system.

\section{Introducción}

El confort de varias salas es uno de los aspectos en constante investigación para el desarrollo de edificios inteligentes (Sierra, 2005). Existen muchas investigaciones centradas en cómo alcanzar o mantener la temperatura de una sala en base al índice PMV (Predicted Mean Vote) (Soyguder, 2009) y otras en desarrollar sistemas que ahorran energía fijando los valores de temperatura y humedad que se quieren mantener (Shahnawaz, 2007). En 2009, Dounis presentó una revisión de los sistemas de control avanzados existentes, basados en el ahorro de energía y la gestión del confort en edificios. En la mayoría de los estudios los controles termales se basaban en el PMV, sobre el que muchos autores son críticos (Van Hoof, 2008).
Es clara pues la necesidad de dotar a estos sistemas de la capacidad de decisión suficiente para actuar sobre el confort ahorrando la mayor cantidad de energía posible. No obstante, existen situaciones para las cuales maximizar el confort debe prevalecer sobre maximizar el ahorro.

El aire acondicionado de un sistema central proviene de un equipo que filtra, calienta o enfría el aire y corrige la cantidad de humedad necesaria. Éste se envía por conductos a los diversos pisos del edificio, extrayéndose una parte del aire viciado e inyectando porciones de aire nuevo mediante manejadores. Pero cuando en la salas se producen peticiones dispares de temperatura es necesario producir centralmente más (o menos) temperatura para que to- 
das las necesidades sean cubiertas. Ajustar el confort para maximizar el ahorro puede suponer una pérdida de la calidad en el confort; en cambio, maximizar el confort durante un periodo de tiempo para permitir la adaptación de los usuarios, esperar y reducirlo a valores de maximización del ahorro puede permitir mejorar la calidad y aceptación de este ahorro. En este sentido, algunos autores muestran estudios en los cuales pequeños aumentos en el porcentaje de personas disconformes (PPD) pueden suponer un ahorro considerable ( 13\%) en la energía consumida (Magnier, 20l0).

Existen situaciones en las cuales debe prevalecer el confort sobre el ahorro energético ya que los usuarios necesitan adaptarse a los cambios de temperatura producidos por la variación en la ocupación del edificio, la baja temperatura corporal en los momentos iniciales del día (Almirall, 1995) tras desayunos y comidas, etc. son hechos que afectan a las sensaciones térmicas humanas que suelen darse en conjunto.

Este artículo pretende mostrar la metodología para identificar patrones de comportamiento que puedan aportar información a considerar por un sistema que tomara decisiones. Se trata de identificar tres estados necesarios en un sistema: Maximizar confort, mantener confort y maximizar ahorro.

\section{Los diseños basados en lógica difusa}

En múltiples aplicaciones de la industria, podemos observar como la lógica difusa es una herramienta basada en la acción, en el caso del confort los usuarios realizan acciones intencionales cuya motivación sólo es conocida realmente por ellos, y que de manera indirecta es conocida por el sistema a partir de un cuestionario de respuestas imprecisas.

El objetivo principal del diseño es proporcionar un sistema de detección fiable pero a la vez de fácil implementación. Esta propuesta metodológica es aplicada porque se desean tomar decisiones que no estén basadas en el conocimiento de actuaciones en el pasado, que otras técnicas como las redes neuronales podrían proporcionar, sino en un instante anterior del espacio temporal.

La búsqueda de patrones situacionales para la obtención de información útil en la toma de decisiones sugiere el empleo de técnicas de inteligencia artificial. La lógica difusa destaca por su sencillez y fácil aplicación y es especialmente adecuada para la con- junción de variaciones de demanda de muy diversas características. Esta técnica consiste en una serie de reglas generales o directrices definidas de forma que el problema que se pretende resolver no se corresponde unívocamente con un modelo determinado de lógica difusa, de ahí su versatilidad en cuanto a implementaciones y rendimiento (Fernández, 2009). Aún así, como es lógico, todo sistema basado en lógica difusa presenta características parecidas: Son sistemas robustos, necesitan poca información de entrada, y su proceso suele constar de las siguientes tres fases: fuzzification (conversión del valor de las variables de entrada en valores "difusos"), proceso de inferencia basado en reglas lógicas; y defuzzification (conversión del valor de las variables difusas y toma de decisión).

Las reglas aplicadas en los sistemas que emplean la lógica difusa son reglas expresadas por los expertos que sólo son aproximadas, pues el modelado del conocimiento de la lógica difusa no es metódico, sino se propone a través de la experiencia del experto.

En la actualidad existe un estándar para implementar lógica difusa, especialmente para el control difuso, denominado FCL (Fuzzy Control Language, IECI | 3 | p7) que facilita el desarrollo junto a librerías de diferentes lenguajes de implementación.

\section{Modelo}

Mediante sensores y gracias a las herramientas tecnológicas actuales (PCs, teléfonos, móviles, PDAs, etc.) se puede personalizar el confort y detectar qué usuarios valoran éste, donde y en que media. Además, se pueden registrar los cambios deseados sobre el estado de las salas.

El objetivo del modelo es proporcionar un sistema fiable y a la vez de fácil implementación, de ahí la sencillez de las entradas requeridas.

\section{I. Parámetro de diseño}

t: Periodo de tiempo tras el cual se realiza el análisis y posterior toma de decisión respecto al tipo de configuración climática.

\subsection{Variables iniciales del modelo.}

Ashrae (1997) define un rango de valoraciones que podría dar un usuario: Calurosa (muy cálida), cálida, ligeramente cálida, neutra, ligeramente fría, fría y muy 
fría. Este rango de valores es muy extenso, teniendo el mismo valor algunas de las componentes en cuanto al patrón que deseamos buscar. Por ello, reducimos este rango a tres componentes que son: Disconforme por calor, conforme y disconforme por frío. Definiéndose las siguientes variables:

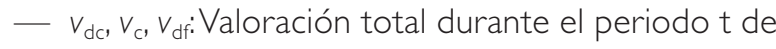
los usuarios. La valoración puede ser: Disconforme por calor, conforme y disconforme por frío.

- $v_{d c}^{\prime}, v_{c}^{\prime}, v_{d f i}^{\prime} V^{\prime}$ ariación de la valoración total durante el periodo t y el periodo $t-1$.

— o: Porcentaje de ocupación en el periodo t.

- o':Variación de la ocupación durante el periodo t y el periodo $t-1$.

Las variaciones de las valoraciones totales durante dos periodos diferentes consecutivos se calculan como:

$$
\begin{gathered}
v_{\alpha}^{\prime} \mathrm{I}_{\Delta t}=\frac{\left.v_{\alpha}\right|_{\Delta t}-v_{\alpha \Delta t-1} \mid}{\left.v_{\alpha}\right|_{\Delta t}} \\
\operatorname{con} \forall \alpha \in\{d c, c, d f\} \\
\left.{ }^{\prime}\right|_{\Delta t}=\frac{{ }^{\left.o\right|_{\Delta t}}-{ }^{\left.o\right|_{\Delta t-1}}}{\left.o\right|_{\Delta t-1}}
\end{gathered}
$$

\subsection{Conversión de las variables a variables difusas (fuzzification)}

Según la posición relativa de las variables definidas previamente respecto de la valoración esperada durante el intervalo, cada variable se divide en términos porcentuales en tres componentes diferentes.

Así, las valoraciones de los usuarios durante el periodo t se representan por tres componentes que las definen, en cuanto a cantidad total se refiere:
- $v_{p}, v_{m}, v_{g}:$ Componente de conjunto pequeño, mediano y grande de usuarios, respectivamente.

En lo que respecta a la variación, tanto en sentido positivo como negativo, del número de usuarios que valoran entre dos periodos consecutivos, también se divide cada parámetro en tres partes o componentes porcentuales según el signo de la variación entre periodos:

- $v_{n}^{\prime}, v_{z}^{\prime}, v_{p}^{\prime}:$ Variación de las valoraciones negativa, nula y positiva respectivamente.

La cuantificación exacta de los componentes de cada una de las variables se rige según las funciones de pertenencia (membership) mostradas en las figuras 1,2 y 3 .

En la Figura I se presenta la función de pertenencia del confort y la variación de éste. La consideración de pertenencia como grupo pequeño en el confort se ha despreciado y en el grupo mediano se ha devaluado. La razón de este cambio se debe a que los usuarios no están obligados a indicar que están en una situación confortable mientras que usan el sistema cuando no hay confort. Téngase en cuenta que si este cambio no se realizase el sistema daría el mismo valor a los disconformes que a los conformes que han decidido responder.

En la Figura I, Figura 2 y Figura 3 la elección del valor que toma como pequeño a un grupo se realiza en base a un porcentaje. En el estudio de Fanger (Ashrae, 1997) se recomienda que no se sobrepase el $10 \%$ de insatisfechos y se sugieren rangos confortables a partir de menos del 20\% de personas insatisfechas. Por tanto son valores que definen lo considerable del tamaño y el sentido de las valoraciones.

También la ocupación total de usuarios durante el periodo t se representa por tres componentes que la definen en cuanto a cantidad total se refiere:

Figura I

Funciones de pertenencia del confort y la variación éste

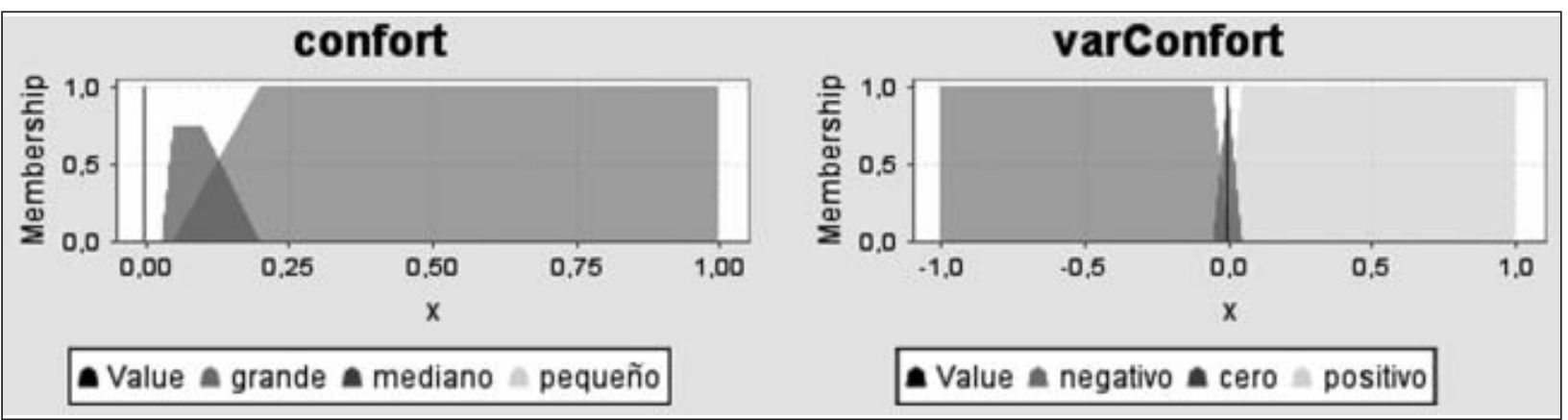

Dirección y Organización, Núm. 42, Diciembre 2010 | ISSN (On line): 2171-6323 - ISSN (Print): II32-I75X 
Figura 2

Funciones de pertenencia cuando no hay confort por calor y la variación éste

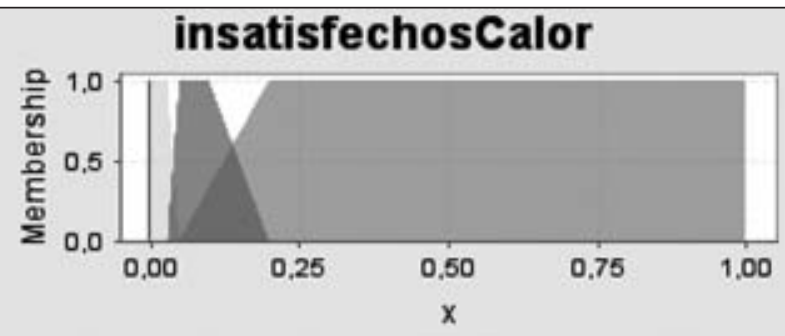

- Value a grande a mediano a pequeño
variCalor

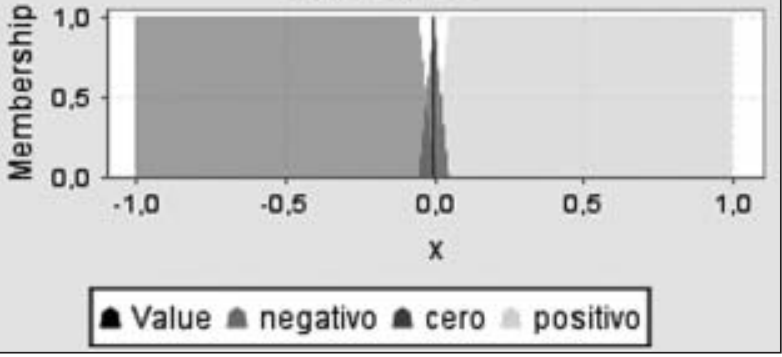

Figura 3

Funciones de pertenencia cuando no hay confort por frío y la variación éste

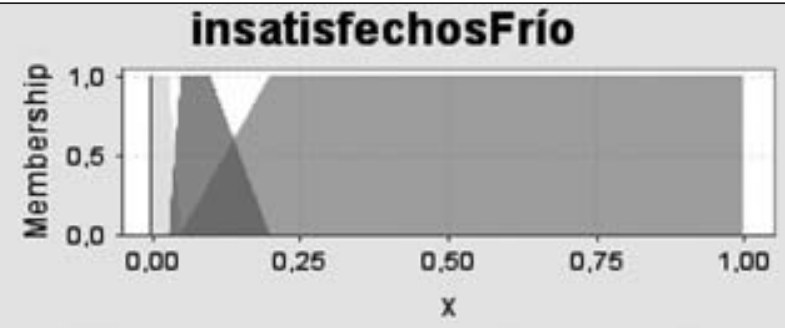

a Value a grande a mediano a pequeño

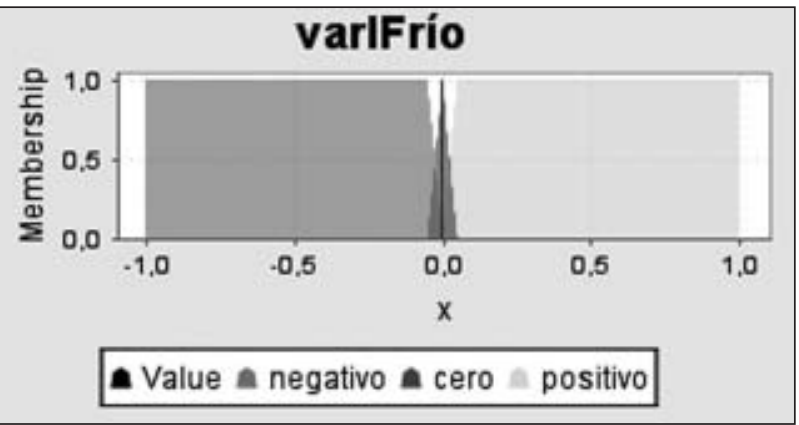

Figura 4

Funciones de pertenencia de la ocupación y la variación ésta

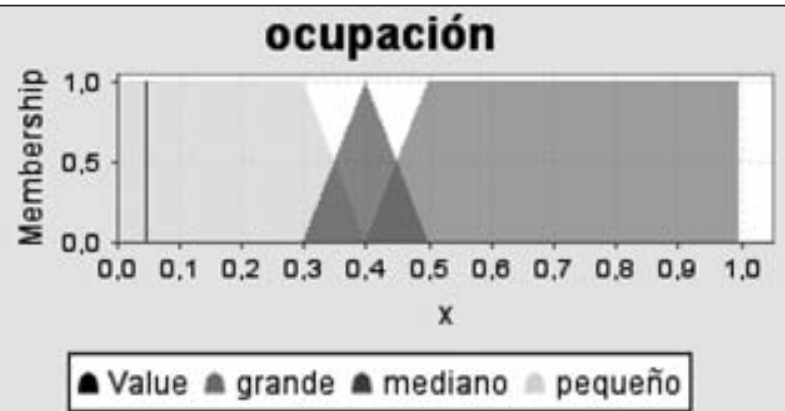

- $\mathrm{o}_{\mathrm{b}}, \mathrm{O}_{\mathrm{m}}, \mathrm{O}_{\mathrm{a}}$ : Componente de ocupación baja, mediana y alta, respectivamente.

En lo que respecta a la variación (tanto ascendente como descendente) de masa de usuarios entre dos periodos consecutivos, también se divide cada parámetro en tres partes o componentes porcentuales según el signo de la variación entre periodos:

- o'n, O' $\mathrm{O}_{\mathrm{p}}$ : Variación de la ocupación negativa, nula y positiva, respectivamente.

La cuantificación exacta de los componentes de cada una de las variables se rige según se muestra en la Figura 4.

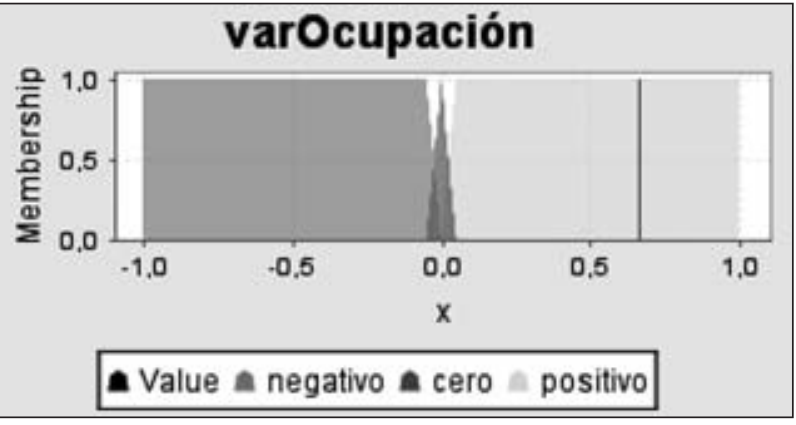

3.4. Proceso de inferencia basado en reglas lógicas

Una vez obtenidas las tres componentes de cada variable, se calcula una serie de reglas lógicas teniendo en cuenta la variación respecto al periodo anterior:

$R /: v_{\text {gdc }} \& \& v^{\prime}{ }_{p d c} \& \& o_{\mathrm{a}} \& \& O_{p}^{\prime}$ entonces Salida $=$ confort $R 2: \ldots$

$\ldots$

La regla $\mathrm{R} I$ indica que se debe dar preferencia al confort si la ocupación del edificio es alta, su varia- 
ción es positiva (están llegando usuarios), el número de usuarios disconformes por calor es grande y su variación es también positiva. En la regla, el producto lógico \&\& representa el mínimo entre los cuatro factores. El significado de confort, neutro y ahorro se utiliza para indicar si existe un cambio considerable del confort a nivel general, y representan la configuración a la que debe de dar preferencia el sistema.

Una vez obtenido el valor de cada regla, se calcula la «fuerza» de las componentes confort, neutro y ahorro mediante la raíz de la suma cuadrática de todas las reglas asociadas a cada una de ellas:

$$
\begin{aligned}
& \text { fuerza } a_{\text {confort }}=\sqrt{\sum_{i} R_{i}} \\
& \forall R_{i} \in \text { confort }
\end{aligned}
$$

\subsection{Conversión del valor de las variables difusas (defuzzification) y toma de decisión}

Para poder tomar una decisión sobre qué demanda debe prevalecer (la de los usuarios o la de ahorrar por parte de un sistema central) es necesario transformar los resultados anteriores del proceso de inferencia en un solo resultado interpretable matemáticamente en forma de probabilidad. Un ejemplo de representación de cada una de las fuerzas se presenta en la Figura 5, que es el resultado tras una variación de crecimiento positivo de la ocupación hasta el $31 \%$, mientras las valoraciones por confort y calor se mantienen a cero, $y$ las valoraciones por insatisfacción por frío aumentan al 6\%, por lo que las reglas dan pertenencia I al confort, 0'87 a neutro y 0'33 en ahorro.

Figura 5

Fuerza de los centros

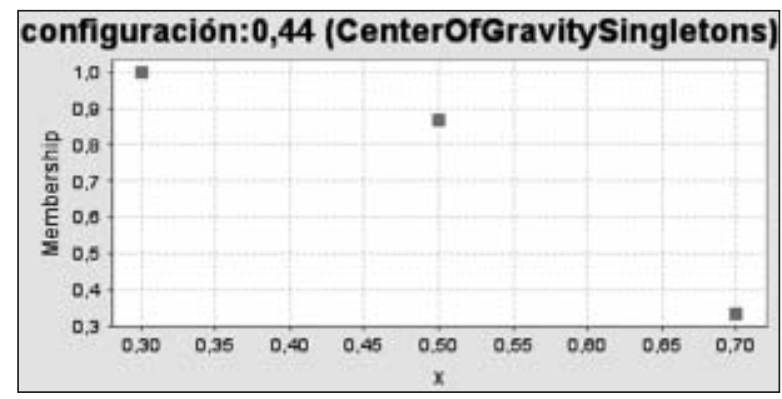

Donde 0'3, 0'5 y 0'7 representan, respectivamente, los «centros» Confort (C), Neutro (N) y Ahorro (A) de las funciones de pertenencia mostradas en la Figura 6.
Figura 6

Funciones de pertenencia

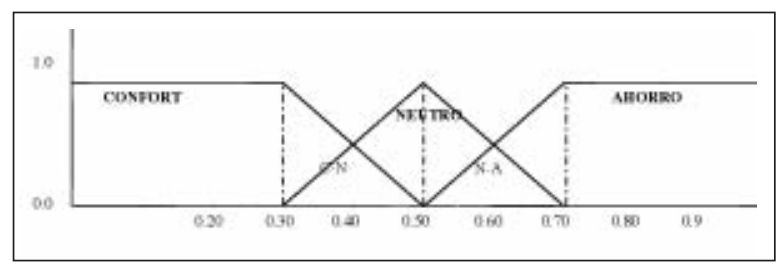

Se pondera cada centro mediante la fuerza de la componente respectiva (calculada anteriormente en el proceso de inferencia) y se calcula la media:

En la Figura 7 se presenta el valor de la solución de la función de pertenencia de salida para el caso mostrado en la Figura 5, se obtiene el centro de gravedad asociado a los comportamientos que se definen; es decir, el punto de referencia respecto a los centros que representan hallarse ante Confort, Neutro o Ahorro. De este punto se deduce el patrón existente en el periodo analizado. Además como no sólo se tiene en cuenta el tamaño del flujo sino la variación de éste respecto al periodo anterior, mediante la elaboración cuidadosa de las reglas, se puede no sólo detectar el patrón de confort sino también predecirlo para el periodo siguiente con una probabilidad de error muy pequeña. Todo ello utilizando la menor cantidad de información posible, habida cuenta de que no se conoce ni la temperatura, ni las particularidades de cada usuario. Las reglas lógicas de decisión, si se detectasen varios tipos, darían como respuesta la de más alta probabilidad.

Figura 7

Salida

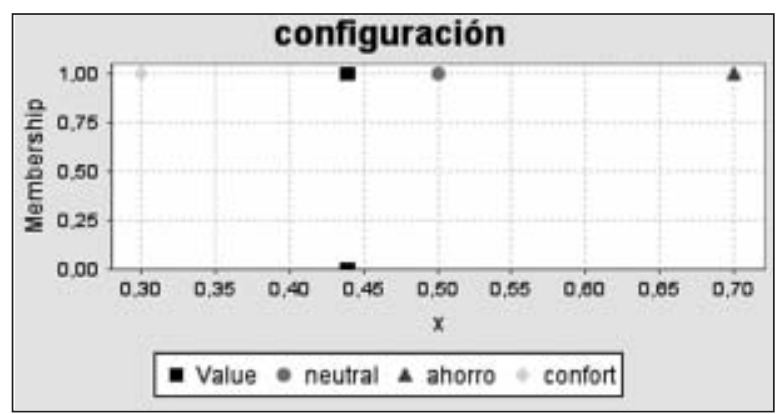

\section{Resultados}

Se ha realizado la simulación de un sistema central de HVAC (Heating, Ventilating and Air-Conditioning) para el área de aproximadamente $800 \mathrm{~m}^{2}$ de un edificio representativo en el que la máxima ocupación 
posible es de cien personas, y que durante el año tiene una ocupación que varía entre el veinte y el sesenta por ciento de ocupación media. Las simulaciones presentadas son para un espacio temporal desde las siete de la mañana (tiempo $h=0$ ) hasta las cuatro de la tarde (tiempo h=9). En la Figura 8 se muestran situaciones sin confort para un día perteneciente a un periodo invernal. La solución se ha representado en un intervalo de cero a uno para mayor claridad.

Figura 8

Cambios de configuración en un periodo invernal con ocupación baja

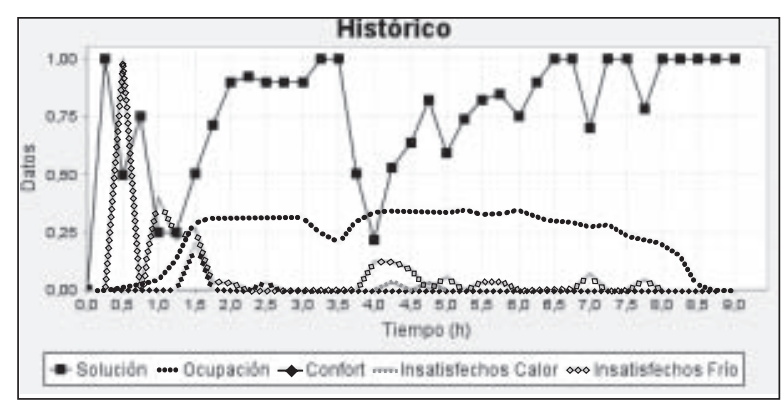

La metodología expuesta ha sido aplicada sobre diferentes simulaciones de datos. En la Figura 8 se muestra la variación de la solución durante nueve horas de estudio. Éstas han sido divididas en espacios temporales de 15 minutos cada una. Estos 36 espacios temporales muestran el estado del edificio en tanto por uno y la configuración que debe ser aplicada (Solución). El valor cero representa la configuración de máximo confort y el valor uno representa el máximo ahorro. En este caso se muestra el edificio con una ocupación baja (entorno al $30 \%$ ). En la figura se observa que el sistema tiende al confort en el cuarto y quinto espacio temporal, debido a que éstos son los primeros instantes del día, y en ellos se observa un crecimiento de la ocupación junto con un alto número de usuarios insatisfechos por frío. En el décimo sexto espacio temporal se observa otra situación similar, esta vez debida a una situación de frío de un grupo importante de usuarios.

En la Figura 9 se puede observar que ante una constante situación de usuarios insatisfechos (durante una hora a partir de la segunda hora) el sistema mantendría la decisión de tomar valores que mejoren el confort.

En las Figura 9 y Figura 10 se muestra el comportamiento ante una ocupación media-alta. En ella se muestra mayor número de personas insatisfechas con la temperatura. En el séptimo espacio temporal se muestra un valor relevante de valoraciones de confort. El valor de estas opiniones es tenido en cuenta por no ser un conjunto pequeño de valoraciones, es por ello que se busca el ahorro pues crecen los conformes y decrecen los usuarios insatisfechos. En cambio, en el décimo primer espacio temporal se muestra una situación para la cual en el edificio en conjunto hay disparidad de sensaciones de confort, unas por frío y otras por calor, por lo que se buscan los valores que permitan el mayor confort del edificio.

Figura 9

Cambios de configuración en un periodo invernal con ocupación media-alta

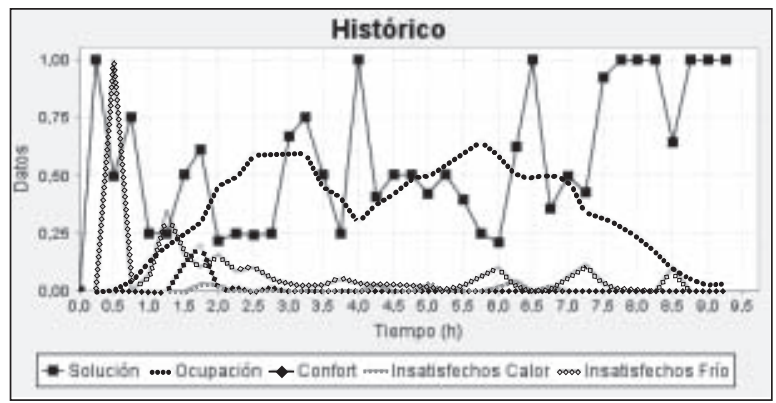

Figura 10

Cambios de configuración en un periodo invernal con ocupación media-alta

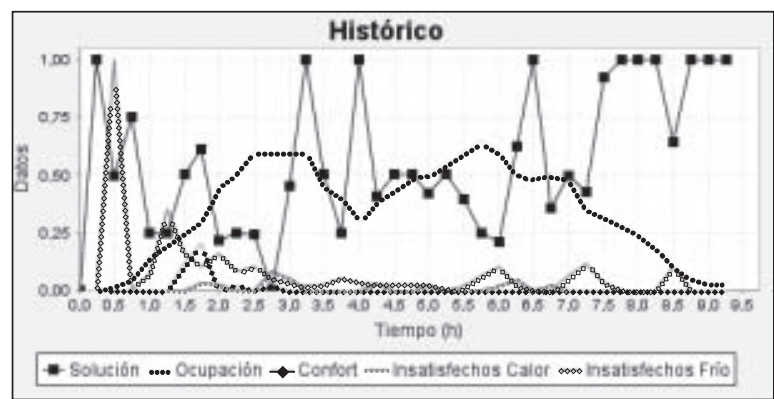

En la Figura I I se muestra una situación en el supuesto de encontrarse en el periodo estival.

La interpretación del resultado puede generar confusión por ser un valor de información aplicable en una toma de decisión. En este sentido, en las figuras Figura 8, Figura 9, Figura I 0 y Figura II un valor uno en la configuración representa el ahorro. Esto significa que el sistema debe de elegir valores dentro del rango de confort que no le supongan esfuerzo ener- 
gético al sistema central. Con certeza el cambio se entiende debe hacerse de manera gradual. Por otro lado, un valor cero representa el confort, esto supone que se deben buscar aquellos valores del rango más centrados, puesto que hay situaciones de confort dispares. Los valores intermedios indican el grado de cercanía a los valores de ahorro y confort. Por ejemplo en el periodo vigésimo de la Figura I I, la solución es aproximadamente 0 '25, lo que supone que el sistema debe dar valores cercanos al máximo confort. Esto es lógico pues los usuarios tienen calor (5\% expresan su insatisfacción) y por tanto el sistema debe generar valores de confort que se alejen del confort que limita con el calor.

Figura | |

Cambios de configuración en un periodo estival con ocupación media-alta

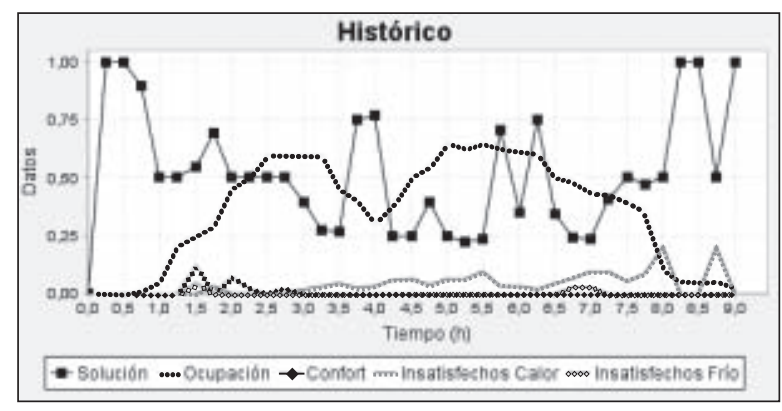

\section{Conclusiones}

En el futuro, los sistemas de HVAC se basarán en preferencias comunicadas por el usuario mediante dispositivos tecnológicos. En las salas de los usuarios los rangos de valores de confort serían conocidos en base a esas preferencias. Los rangos de valores pueden ser amplios o no, pero no siempre se pueden elegir los valores de máximo ahorro, al igual que en otros estudios tomábamos el porcentaje de personas disconformes, en éste se presenta la metodología que permite tomar la decisión sobre el valor del rango a elegir en función de la situación del sistema central, lo que permitiría a éste ahorrar y relajar el valor de confort en situaciones en que éste es generalizado por la adaptación de los usuarios. También en situaciones del día en que el malestar es general permitiría detectar la situación y maximizar el confort de los usuarios en cuanto a la capacidad permitida por el sistema central.

Los cambios que ocurren dentro de un periodo de tiempo no son detectados. El periodo de tiempo elegido puede ser modificado, al igual que otros pará- metros que deberían de ser calibrados para el uso en un sistema real, como ocurre de manera general en los sistemas de lógica difusa.

La metodología aplicada ofrece información que permite la toma de decisiones necesaria para lograr el mayor número de usuarios conformes y el ahorro gradual de la energía en los periodos en los que los usuarios estén adaptados. Esta metodología permite un mayor ahorro frente a la adaptación a situaciones climáticas fijas que se utilizan en los sistemas actuales.

\section{Referencias}

I525I UNE-EN (2008). Parámetros del ambiente interior a considerar para el diseño y la evaluación de la eficiencia energética de edificios incluyendo la calidad del aire interior, condiciones térmicas, iluminación y ruido. AENOR

ALMIRALL H.; MARCET, C. (1995). Evolución de la temperatura corporal a lo largo del día, función de crecimiento y cronotipo. Psicothermal,Vol. 7, No. 2, pp. 317 326.

ASHRAE (1997). Ashrae Handbook-Fundamentals - Atlanta. American Society of Heating, Refrigerating and AirConditioning Engineers, Inc.

DOUNIS, A.I.; CARAISCOS, C. (2009). Advanced control systems engineering for energy and comfort management in a building environment - A review. Renewable and Sustainable Energy Reviews, 13, pp. |246-1261.

FERNÁNDEZ, J.R., CORTÉS, P.; DELGADO, M.C. (2009). Controlador basado en lógica difusa para la detección del patrón de tráfico en sistemas de transporte vertical. Barcelona-Terrassa. 3rd International Conference on Industrial Engineering and Industrial Management.

INTERNATIONAL ELECTROTECHNICAL COMMISSION (IEC). "IEC I I I Programable Controllers. Part 7: Fuzzy Control Programming”. IEC TC65/WG 7/TF8. 1997

MAGNIER, L.; HAGHIGHAT, F. (20I0). Multiobjective optimization of building design using TRNSYS simulations, genetic algorithm, and artificial neural network. Building and Environment, 45, pp. 739-746.

SHAHNAWAZ, S. (2007). Fuzzy logic based energy saving technique for a central air conditioning system. Energy, 32, pp. $1222-1234$.

SIERRA E. A.; HOSSIAN, A. A.; MARTÍNEZ, R. G.; MARINO, P. D. (2005). Sistema experto para control inteli- 
gente de las variables ambientales de un edificio energéticamente eficiente. XI Reunión de Trabajo en Procesamiento de la Información y Control.

SOYGUDER, S.; ALLI, H. (2009). An expert system for the humidity and temperature control in HVAC systems using ANFIS and optimization with Fuzzy Modeling Approach. Energy and Buildings, 4I, pp. 8I4-822.

VAN HOOF, J. (2008). Forty years of Fanger's model of termal confort: Comfort for all?. Indoor Air, I8, pp. I82201. 